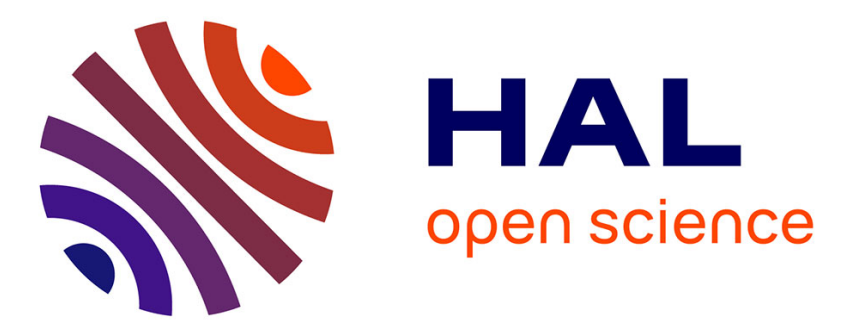

\title{
Subwavelength metallic coaxial waveguides in the optical range: Role of the plasmonic modes
}

\author{
F.I Baida, A. Belkhir, D. van Labeke, O. Lamrous
}

\section{To cite this version:}

F.I Baida, A. Belkhir, D. van Labeke, O. Lamrous. Subwavelength metallic coaxial waveguides in the optical range: Role of the plasmonic modes. Physical Review B: Condensed Matter and Materials Physics (1998-2015), 2006, 74 (20), pp.205419. 10.1103/PhysRevB.74.205419 . hal-00258571

\section{HAL Id: hal-00258571 https://hal.science/hal-00258571}

Submitted on 18 Apr 2021

HAL is a multi-disciplinary open access archive for the deposit and dissemination of scientific research documents, whether they are published or not. The documents may come from teaching and research institutions in France or abroad, or from public or private research centers.
L'archive ouverte pluridisciplinaire HAL, est destinée au dépôt et à la diffusion de documents scientifiques de niveau recherche, publiés ou non, émanant des établissements d'enseignement et de recherche français ou étrangers, des laboratoires publics ou privés. 


\title{
Subwavelength metallic coaxial waveguides in the optical range: Role of the plasmonic modes
}

\author{
F. I. Baida,* A. Belkhir, and D. Van Labeke \\ Département d'Optique P. M. Duffieux, Institut FEMTO-ST UMR 6174 CNRS, Université de Franche-Comté, \\ 25030 Besançon Cedex, France \\ O. Lamrous \\ Laboratoire de Physique et Chimie Quantique, Université Mouloud Mammeri, Tizi-Ouzou, Algeria \\ (Received 11 July 2006; revised manuscript received 25 September 2006; published 16 November 2006)
}

\begin{abstract}
A theoretical investigation is presented of the mode propagation and attenuation in a nanometric coaxial waveguide in real metal. By a rapid comparison with other structures, it is established that a coaxial waveguide has propagative modes with very interesting properties: the cutoff wavelengths are very large, they become larger when a perfectly electric conductor is replaced by gold or silver (real metal), and they can be increased when the outer and inner radii are very close one to other. By studying dispersion curves and field structures, it is shown that surface plasmon modes are responsible for these properties. By simply changing the geometrical parameters of the structure, a very large effective index and very low group velocities could be obtained. We also establish that, in spite of the metal losses, a reasonable large propagation length could be obtained $(50 \mu \mathrm{m})$ which should allow applications for guiding light in nano-optics.
\end{abstract}

DOI: 10.1103/PhysRevB.74.205419

PACS number(s): 78.67.-n, 78.20.Bh, 42.79.Gn

Miniaturization of optical components is now a real challenge and takes a large place in the domain of nanotechnology. New optical devices are currently designed at the micrometer scale. Tunable photonic crystals are a significant example of such devices. ${ }^{1}$ Surface plasmons, which are localized electromagnetic waves, are used to enhance singlemolecule fluorescence ${ }^{2}$ or to build microdetectors for biological applications. ${ }^{3}$ The superoptical transmission obtained when light passes through a metallic array of subwavelength holes, ${ }^{4-6}$ is now used as a detector for organic molecules and could be useful to modulate, to filter or to polarize light at nanometric scales. ${ }^{6,7}$ Motivated by transmission problems through optical near-field probes, some theoretical studies were performed on metallic nanometric cylindrical guiding structures. $^{8,9}$ Waveguides that can transmit both electrical and optical signals should also be designed at this scale as proposed in Ref. 10. In their paper, Bozhevolnyi et al. clearly demonstrate that a simple groove on a metallic layer can play the role of an effective waveguide for surface plasmons with a relatively very large decay length.

The aim of our paper is to propose another solution for optical connections in nano-sized optical components: we theoretically establish that a coaxial waveguide with submicrometer radii could guide light with a much larger wavelength and with losses low enough to enable useful propagation distances.

In order to use light $(0.4<\lambda<1.5 \mu \mathrm{m})$ for optical connections in nano-optical components, it is necessary to find a structure with small transverse widths and with losses low enough to enable a useful propagation distance. Dielectric materials generally have negligible losses but the transverse confinement is very difficult to obtain with such materials. Some photonic optical fibers have subwavelength channels but the field is delocalized among all the cross sections of the fiber. A dielectric fiber can be easily tapered at a nanometric scale and it can guide light without any cutoff restriction. But the evanescent part of the guided mode is spread out over the taper, which is not compatible with efficient confinement.

With metallic guides, at first glance, it seems easier to confine the field in a transverse plane, but it is then necessary to seriously discuss the problems of wavelength cutoff and of losses especially if real metals are used. The properties of metallic waveguides can be found in many textbooks (Ref. 11, for instance), but the studies are generally restricted to radio waves or microwaves. For theses spectral domains, the metals are very close to perfect electric conductors (PECs) and losses are treated as a small perturbation. In the optical range, the properties of the metals must be described by a complex dielectric constant with dispersion and losses. For noble metals (silver, gold, aluminum), experimental tables are published $^{12}$ and a good approximation is given by the Drude model $\varepsilon(\omega)=1-\omega_{p}^{2} /\left(\omega^{2}+i \omega \tau\right)$, where $\omega_{p}=1.374$ $\times 10^{16} \mathrm{rad} / \mathrm{s}$ is the plasma frequency and $\tau=3.21$ $\times 10^{13} \mathrm{rad} / \mathrm{s}$ a coefficient directly related to the losses. It is important to notice that for those metals, the losses remain small and the imaginary part of the dielectric constant is much smaller than its real part.

For a guiding structure along the $z$ axis, the electric field of a mode can be written in the general form

$$
\vec{E}=\vec{E}_{0}(x, y) e^{i(\gamma z-\omega t)}=\vec{E}_{0}(x, y) e^{i \omega\left(n_{e f f} / c z-t\right)} .
$$

The propagation coefficient $\gamma$ is directly related to the effective index of the mode: $\gamma=n_{\text {eff }} \omega / c$. In general, $\gamma$ and $n_{e f f}$ are functions of $\omega$ and they depend upon the geometry and on the mode. Without losses, $\gamma$ is real or purely imaginary. For a propagative mode, $\gamma$ is real; for a nonpropagative evanescent mode it becomes purely imaginary. The cutoff frequency corresponds to the limit between the two kinds of modes. At this limit, $\gamma$ and the effective index vanish. For real metals with losses, $\gamma$ is complex and a general discussion is very difficult. However, when losses are small, a clear 


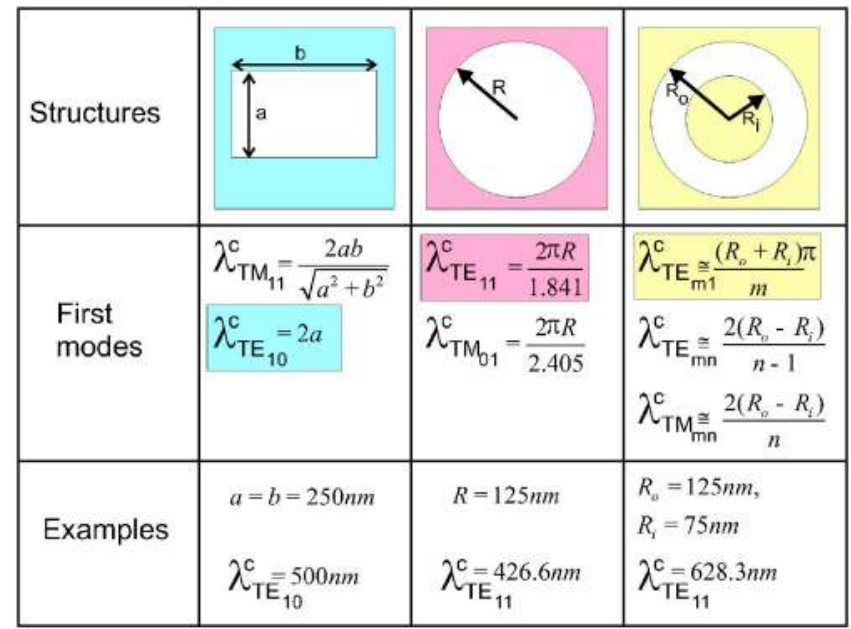

FIG. 1. (Color online) Cutoff wavelengths of the first guided modes (associated with bigger values of wavelength) for three different waveguides made in a PEC.

difference between propagative and attenuated modes remains valid: for propagative modes $\operatorname{Im}(\gamma) \ll \operatorname{Re}(\gamma)$ and a cutoff frequency still exists when $\operatorname{Re}(\gamma) \approx 0$.

To propagate light in a guide with a small cross section, it is necessary to find the geometry that has the mode with the largest cutoff wavelength. Figure 1 gives the cutoffs of the fundamental modes of three kinds of waveguide made with PECs. In the following, the cavity inside each guide is supposed to be filled with air. In theoretical calculations it is easy to replace air by another dielectric; it is not so easy experimentally. So, in the following all the calculations are performed with air inside the cavities.

For a square or rectangular waveguide (widths $a$ and $b$ with $a \geqslant b$ ) in a PEC, the fundamental mode with the largest cutoff wavelength is the $\mathrm{TE}_{10}: \lambda^{c}=2 a$. By replacing a rectangular cross section by a circular one, with the same width (diameter $a=2 R$ ), a smaller cutoff is obtained for the fundamental mode: $\lambda^{c}=1.7 a$ for the $\mathrm{TE}_{11}$ mode.

If we consider a coaxial waveguide, in a PEC, a very interesting result is obtained. The cutoff wavelength of all the modes except two depends on the difference between the outer and inner radii, i.e., the wavelength cutoff is very small. But the $\mathrm{TEM}_{0}$ mode has no cutoff $\left(\lambda^{c}=\infty\right)$ and the $\mathrm{TE}_{m 1}$ mode has a cutoff proportional to the sum of the radii: $\lambda_{T E_{m 1}}^{c} \simeq \pi\left(R_{o}+R_{i}\right) / m$, where $R_{o}$ and $R_{i}$ are the outer and inner radii, respectively. The $\mathrm{TEM}_{0}$ mode has a cylindrical symmetry and it should be very difficult to be optically produced. On the contrary the electric field of $\mathrm{TE}_{11}$ is linearly polarized and could be easily excited.

Thus, except for the $\mathrm{TEM}_{0}$ mode, the fundamental mode of a coaxial guide in a PEC is the $\mathrm{TE}_{11}$ mode, which has a rather large cutoff wavelength: $\lambda_{T E_{11}}^{c} \simeq \pi\left(R_{o}+R_{i}\right)$. If the external transverse width of the coaxial waveguide remains constant, the cutoff wavelength of the $\mathrm{TE}_{11}$ mode can be increased when the inner radius tends to the external one. The maximum that can be reached is $\lambda_{T E_{11}}^{c} \rightarrow \pi\left(2 R_{o}\right)$ which is obtained for a very small gap between the two radii.

For optical applications, it is necessary to check if the

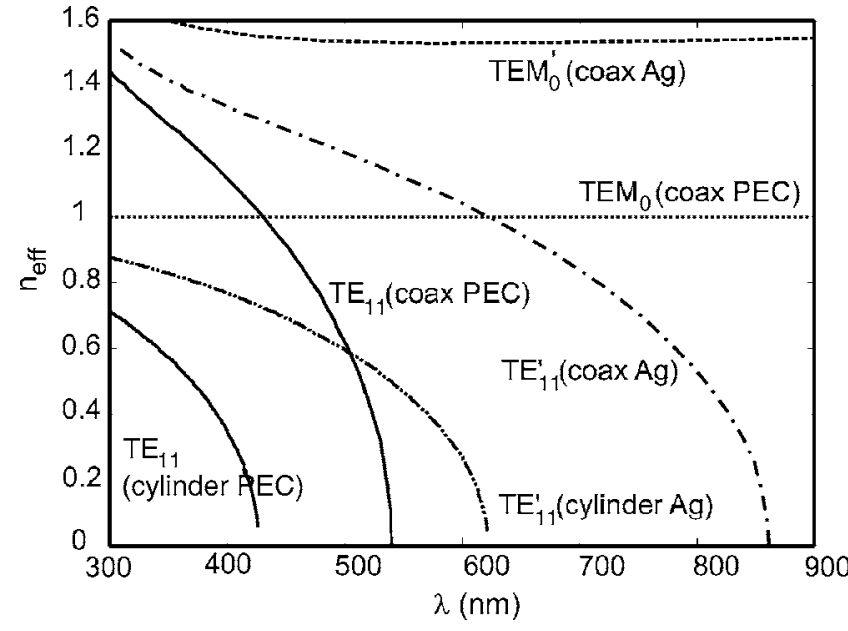

FIG. 2. Effective index of the first guided modes for coaxial and cylindrical waveguides made in PEC and in silver. The radius of the cylindrical waveguide is set to $R=125 \mathrm{~nm}$, the outer radius of the coaxial waveguide is $R_{o}=125 \mathrm{~nm}$, and the inner one is $R_{i}=75 \mathrm{~nm}$.

interesting properties of the coaxial waveguide remain valid in the optical domain. Here analytical solutions are not possible and it is necessary to use numerical methods. Commercial codes are available for this purpose; they are founded upon the finite-difference time domain (FDTD) method or the finite-element method (FEM). They have no problems for dielectric structures but they have shown erratic problems of convergence when applied to our nanoguiding structures with real metals.

The cutoff frequencies and the light distribution inside the waveguide, which will be shown below, are calculated via an original FDTD code that will be briefly described here. The study of axially symmetrical structures can be easily done by the body-of-revolution FDTD (BOR FDTD) method which is based on the discretization of the Maxwell equations expressed in cylindrical coordinates. ${ }^{13,14}$ The $N$-order FDTD method $^{15,16}$ is then adapted to such a symmetry. To our knowledge, this method is not yet recognized in cylindrical

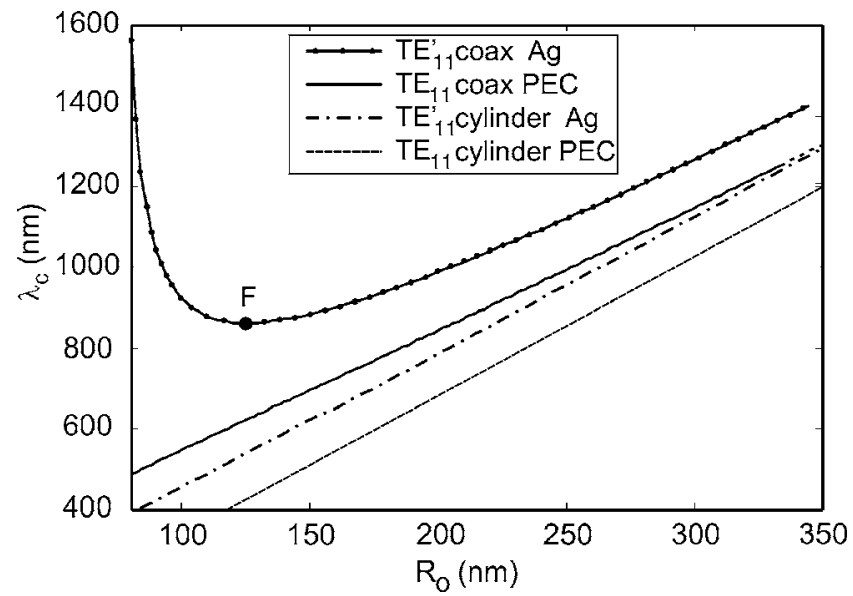

FIG. 3. Variations of the cutoff wavelength of the first mode of the two structures (coaxial and cylindrical) versus outer radius. For the coaxial waveguide, the inner radius is set to $R_{i}=75 \mathrm{~nm}$. 

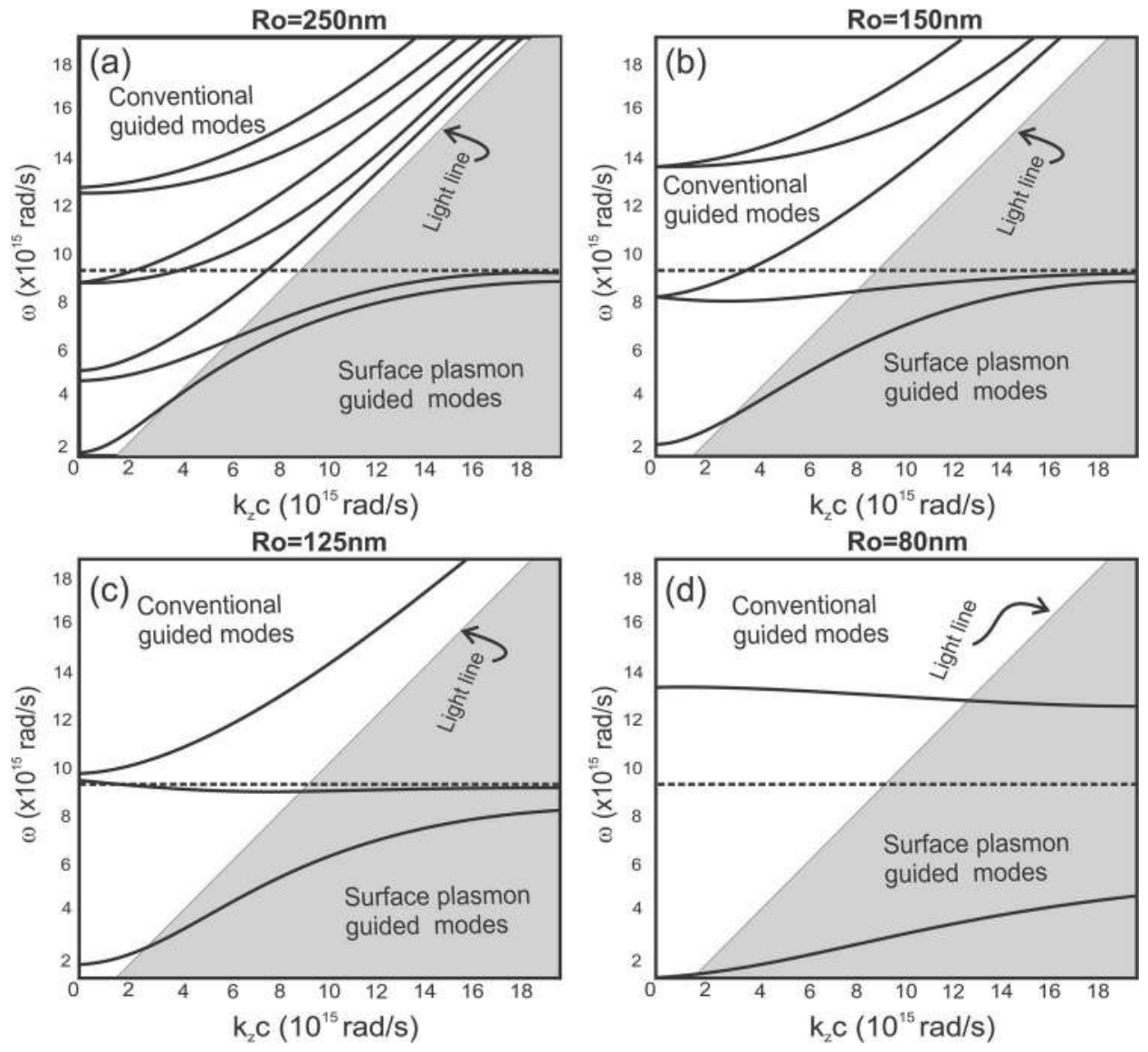

FIG. 4. Dispersion curves of a silver coaxial waveguide with four values of $R_{o}$ and with $R_{i}=75 \mathrm{~nm}$. Only the modes with $m=1$ are shown. The dashed line presented on the four figures corresponds to $\omega=\omega_{p} / \sqrt{2}$. coordinates; however, it leads to an efficient and fast code as it avoids the use of absorbing boundary conditions except in the radial direction. Moreover, we have also used for these calculations, a nonuniform mesh: the smallest spatial step was $\delta_{r}=0.1 \mathrm{~nm}$ near the edges whereas $\delta_{r}=5 \mathrm{~nm}$ elsewhere.

Let us notice here that studies on optical fibers by the FDTD method are generally performed with a twodimensional 2D algorithm with rectangular discretization ${ }^{17,18}$ which is not very well adapted to efficiently describe the cylindrical geometry of the studied structure.

In our case, the dispersion of metals at optical frequencies is easily incorporated in the FDTD algorithm via the discretization of the constitutive equation of the medium that connects the displacement $\vec{D}$ vector to the electric field $\vec{E} .19$

In order to test the validity of our approach, we first determine the dispersion curve for a coaxial waveguide in a PEC and compare the result with the same structure in silver. An example of the dispersion curves is presented in Fig. 2 where are plotted the variations of the effective indices of the first modes $\left(\mathrm{TEM}_{0}, \mathrm{TE}_{10}\right.$, and $\left.\mathrm{TE}_{11}\right)$ versus the vacuum wavelength for two different geometries.

The dispersion curves of the PEC structure, obtained with our FDTD code, exactly correspond to the theoretical ones. The $\mathrm{TEM}_{0}$ mode has no cutoff and its effective index is equal to 1 along the whole spectrum. The effective index of the $\mathrm{TE}_{1}$ mode verifies the simple equation $n_{\text {eff }}^{P E C}(\omega)$ $=\sqrt{1-\lambda^{2} /\left(\lambda^{c}\right)^{2}}$.

For the coaxial waveguide in silver, only two modes have a propagative part in the studied spectral range. A mode with cylindrical symmetry is found, its cutoff wavelength far beyond the infrared, and the effective index exhibits a small dispersion from $\lambda_{0}=600-900 \mathrm{~nm}$ (even up to $1600 \mathrm{~nm}$ ). It is important to notice that the effective index of this mode is larger than 1 . The other mode has a field distribution corresponding to an $m=1$ mode. The two modes have, in the case of silver, small $E_{z}$ field components so they are not pure $\mathrm{TEM}_{0}$ and $\mathrm{TE}_{1}$ modes, but they are in the continuity of the previously described modes of the PEC structure and they will be named in the following the $\mathrm{TEM}_{0}^{\prime}$ and $\mathrm{TE}_{11}^{\prime}$ modes.

The $\mathrm{TE}_{11}^{\prime}$ mode has a dispersion curve which looks like the PEC one but is pushed towards the red region of the spectrum. For a real noble metal, the cutoff wavelength is increased compared to the same structure in the PEC. A similar result has also been recently established for the mode propagation in rectangular ${ }^{20}$ or cylindrical ${ }^{18}$ structures made of real metals. So a general property can be expressed: for a guiding structure made in real metal, the cutoff wavelength of a propagative mode is increased when compared with the same mode of the same structure made in a PEC. This result is a key point for the interpretation of the very large transmission obtained with an annular aperture array. ${ }^{16}$ The redshift depends both on the value of the plasma frequency $\left(w_{p}\right)$ used in the Drude model and on the geometrical parameters of the waveguide (radii). Moreover, the loss coefficient $\tau$ of the permittivity has a very small influence on the cutoff shift.

It is important to notice that, among the three studied structures with the same external radius, the coaxial waveguide in silver is the structure which has propagative modes with the largest wavelength. 

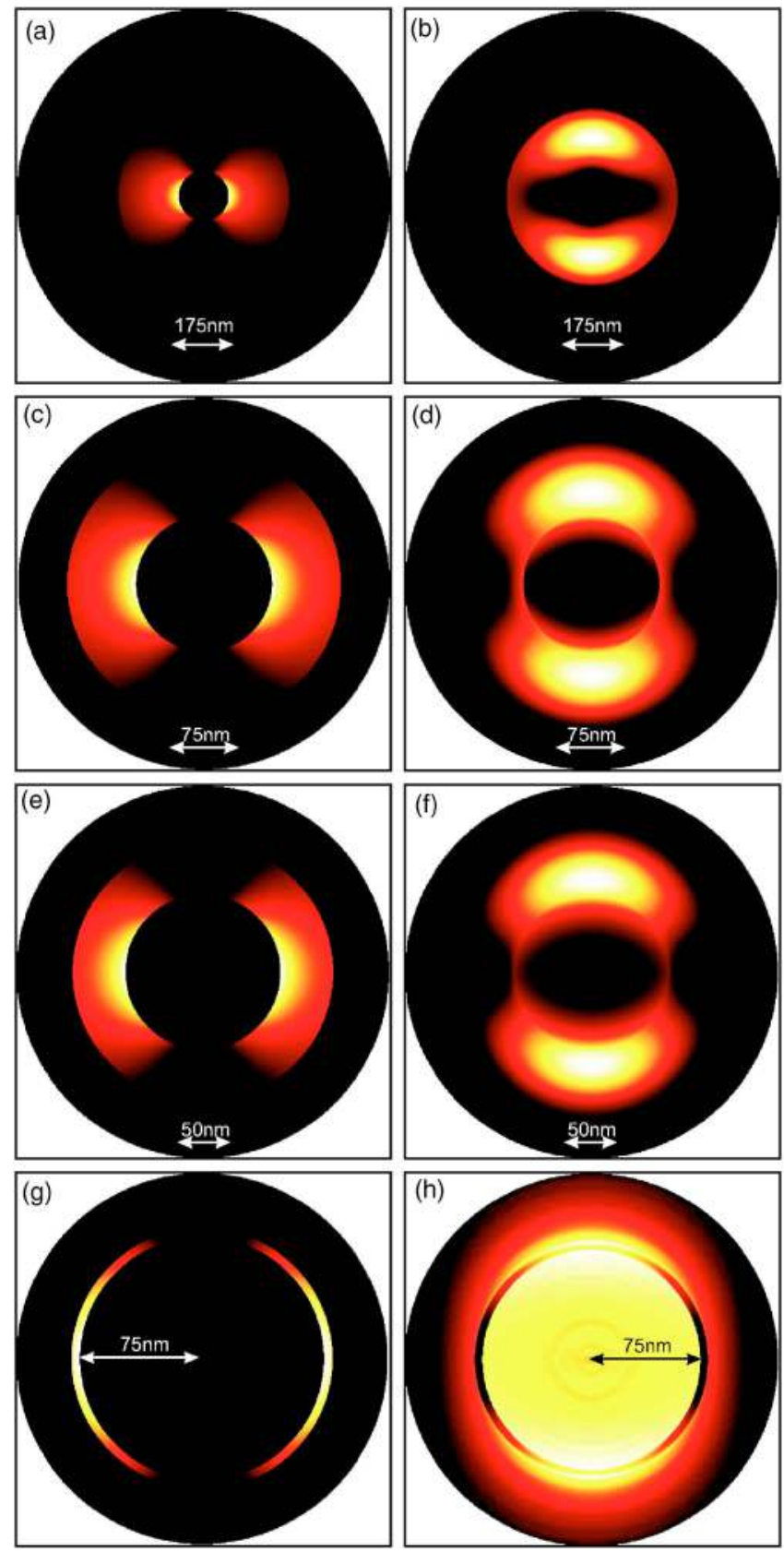

FIG. 5. (Color online) Light distributions (square modulus of the electric field) in a section of the waveguide for four values of $R_{O}$ and with $R_{i}=75 \mathrm{~nm}$. In all the eight subfigures, $k_{z}$ is set to zero, i.e., $\lambda$ is equal to $\lambda^{c}$. (a), (c), (e), and (g) correspond to the lower mode while (b), (d), (f), and (h) are calculated for the second plasmonic mode.

Another interesting phenomenon is presented in Fig. 3. In this figure we have plotted the variations of the cutoff wavelengths versus the outer radius $R_{o}$ of the fundamental modes for four structures: a coaxial waveguide in PEC or silver and a cylindrical waveguide also in PEC or silver. For the two coaxial waveguides, the inner radius is fixed to $R_{i}=75 \mathrm{~nm}$.

For a cylindrical waveguide made in PEC or silver, the behavior of the cutoff is linear but it is shifted toward the red region of the spectrum in the case of real metal. We have also

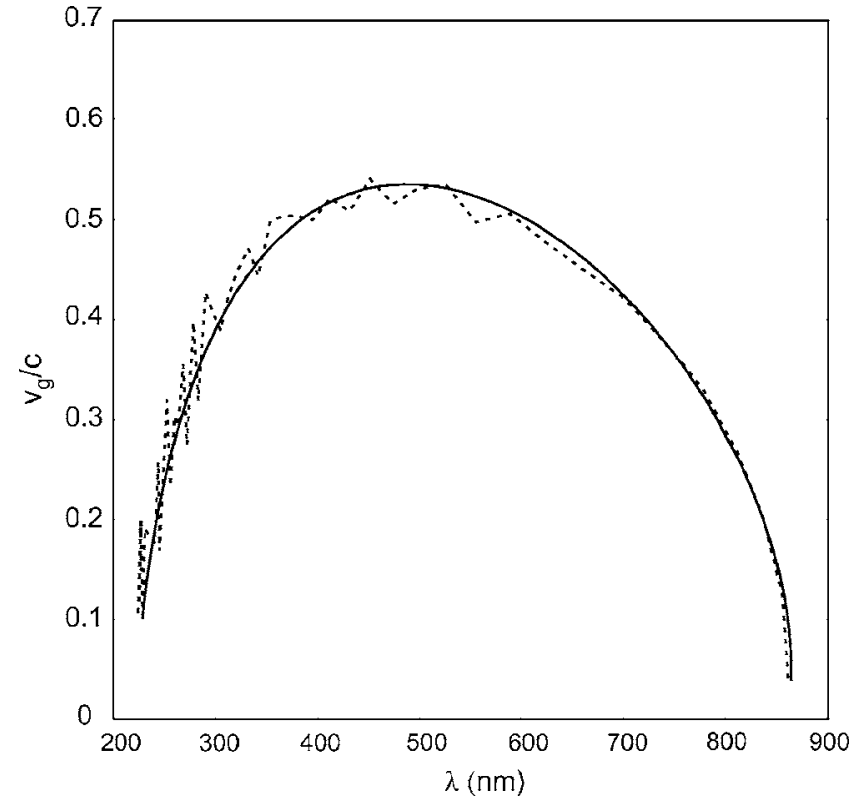

FIG. 6. Normalized group velocity (by c) of the lower mode versus the wavelength for $R_{o}=125 \mathrm{~nm}$ and $R_{i}=75 \mathrm{~nm}$.

reproduced the result presented in the inset of Fig. 2(a) by Shin et $a l .{ }^{18}$

The cutoff wavelength of the $\mathrm{TE}_{11}$ mode of the coaxial waveguide made in the PEC varies linearly with respect to the outer radius and verifies the theoretical equation $\lambda_{T E_{11}}^{c}$ $\approx \pi\left(R_{o}+R_{i}\right)$. All these results are tests showing the good accuracy of our FDTD code.

But for a coaxial waveguide made in silver (metal with losses) one can see in Fig. 3 that the variation of the cutoff wavelength of the $\mathrm{TE}_{11}^{\prime}$ mode exhibits an unusual behavior when the external radius decreases. For a large external radius, the cutoff wavelength first decreases linearly, but, below a limiting value (point $F$ on the figure) $R_{o}^{\min }=125 \mathrm{~nm}$, the cutoff wavelength increases when $R_{o}$ decreases. This finding is at the origin of the enhanced transmission obtained through annular aperture arrays with a small gap between $R_{o}$ and $R_{i}$ when illuminated by very large incident wavelengths. ${ }^{21}$

In order to explain this unusual behavior of the cutoff wavelength in the case of a coaxial waveguide in real metal, we study the evolution of the dispersion curves when $R_{o}$ varies and for a fixed value of $R_{i}=75 \mathrm{~nm}$. The calculations are performed only for modes having the same azimuthal number $m=1$.

Figure 4 shows the results obtained for only four values of $R_{o}$ around the point $F$ of Fig. 3. The higher modes are propagative ones and, as usual, they tend asymptotically to the light line for large values of $k_{z}$. For small values of $R_{o}$, these modes are shifted toward high frequencies; they do not appear in Fig. 4(d) for $R_{o}=80 \mathrm{~nm}$.

The two interesting modes are the lowest ones. Actually, they cut the light line and tend asymptotically to $\omega=\omega_{p} / \sqrt{2}$ which is the surface plasmon frequency on a flat metalvacuum interface. When $R_{o}$ decreases, the two curves repels each other and a large gap is obtained for $R_{o}=80 \mathrm{~nm}$. These 


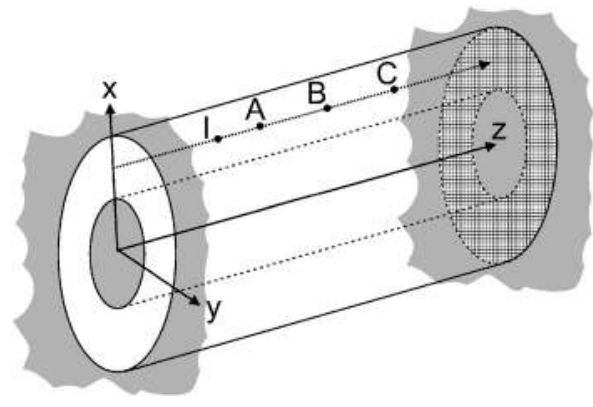

FIG. 7. Schema of the studied coaxial waveguide. A pulsed $\mathrm{TE}_{11}$ guided mode is injected at $z=z_{I}$. Light intensities are then recorded at $z=z_{A}, z_{B}$, and $z_{C}$.

two modes look like the two plasmonic modes obtained in the case of a $1 D$ metallic photonic crystal. ${ }^{22}$ In the former case, one of them has an odd symmetry while the other presents an even one. In our case, there is no geometrical symmetry in the radial direction except when $R_{o} \rightarrow R_{i}$; this implies that there are no even or odd symmetries. Figure 5 shows the light distribution at cutoff wavelengths for the two lower modes in the case of a coaxial waveguide made in silver with $R_{i}=75 \mathrm{~nm}$ and for the four values of $R_{o}$ considered in Fig. 4. One can see that for the fundamental mode, the light distribution corresponds to the excitation of a surface plasmon on the inner interface whichever the value of the outer radius. Moreover, for the second mode, the maxima of light are located between the two interfaces. We have verified that these two modes are $\mathrm{TE}_{1 n}$-like ones, i.e., at the cutoff, $E_{r}, E_{\phi}$, and $H_{z}$ are not equal to zero while the three other components $\left(E_{z}, H_{r}, H_{\phi}\right)$ of the electromagnetic field are null.

Note here that for an outer radius of $R_{o}=80 \mathrm{~nm}$ [Fig. $5(\mathrm{~h})]$, the cutoff wavelength of the second mode is almost equal to $\lambda^{c}=140 \mathrm{~nm}$ which corresponds to $\omega=\omega_{p}$. In this case, the dielectric constant of silver becomes null and a plasmon resonance (in the volume) is obtained as shown in Fig. 5(h).

It seems that, when $R_{o} \rightarrow R_{i}$, the cutoff frequency tends to zero for the fundamental mode and to $\omega_{p}$ for the second plasmonic mode. It would be interesting to have a theoretical interpretation of this phenomenon.

Figure 4(d) shows also that, for a small value of the outer radius (here $R_{o}=80 \mathrm{~nm}$ ), the dispersion curves become almost horizontal. The group velocity $v_{g}$ is then small compared to $c$ (light velocity in vacuum). Figure 6 shows the group velocity versus the wavelength of the lowest guided mode in the case of a silver coaxial waveguide with $R_{o}$ $=125 \mathrm{~nm}$ and $R_{i}=75 \mathrm{~nm}$. This curve is simply numerically calculated from Fig. 4(c) by $v_{g}=d \omega / d k$. We have performed many other calculations which demonstrate that for $R_{o} \rightarrow R_{i}$ $\rightarrow 0$, the dispersion curve becomes more flat and, consequently, the group velocity decreases (for example with $R_{O}$ $=55 \mathrm{~nm}$ and $R_{i}=50 \mathrm{~nm}$, we get $v_{g}<c / 4$ for the whole visible region). In all cases, the value of $v_{g}$ falls to zero at the cutoff because there is no propagation along the $z$ axis $\left(k_{z}=0\right)$.

These modes are interesting because of their large cutoff wavelengths (especially the lowest one). Nevertheless, for

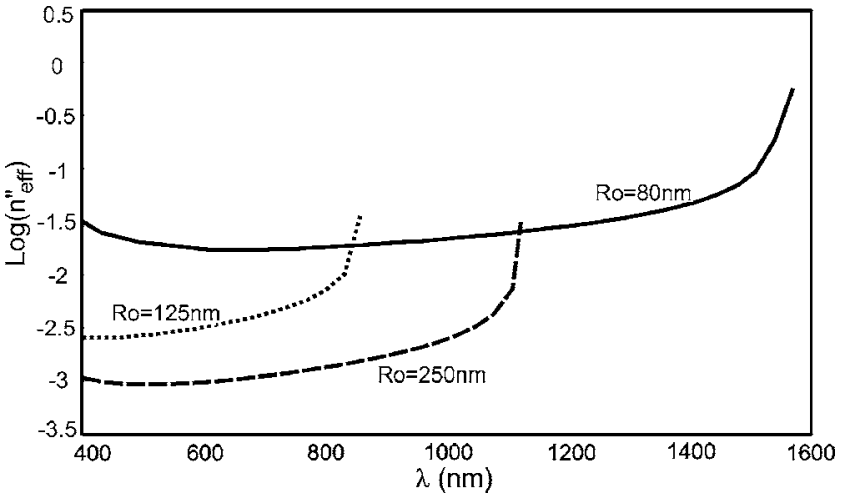

FIG. 8. Numerical values of the imaginary part of the effective index for the $\mathrm{TE}_{11}^{\prime}$ mode in the case of a silver coaxial waveguide with $R_{i}=75 \mathrm{~nm}$. Distances $A B$ and $B C$ were set to $z_{B}-z_{A}=z_{C}-z_{B}$ $=500 \mathrm{~nm}$ and the injection point $I$ was located at $500 \mathrm{~nm}$ above $A$ (see Fig. 7).

practical applications, it is necessary to determine the propagation losses. It is clear from Fig. 5 that, for the fundamental mode $\left(\mathrm{TE}_{11}^{\prime}\right)$, light is essentially confined in the gap between the two metallic parts of the waveguide. This indicates that losses should be very weak during the propagation.

In order to confirm that, let us determine the imaginary part of the effective index $\left(n_{e f f}=n_{e f f}^{\prime}+i n_{e f f}^{\prime \prime}\right)$. The real part $n_{e f f}^{\prime}$ (which is presented in Fig. 2) is determined from the dispersion curves given in Fig. 4 by $n_{e f f}^{\prime}=\lambda^{c} k_{z} / 2 \pi$. The imaginary part of $n_{\text {eff }}$ can be determined by studying the width of the resonance peaks obtained by the $N$-order FDTD method. ${ }^{23}$ But this method is not sufficiently accurate in our case. Thus, we have determined it by performing a numerical propagation experiment. A body-of-revolution FDTD calculation on

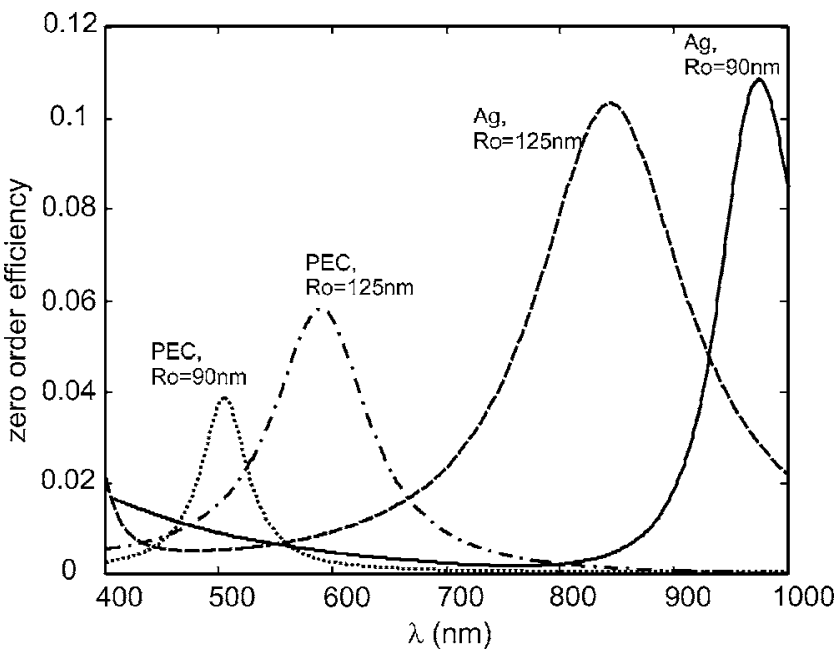

FIG. 9. Comparison of the efficiency of the diffracted zero order by four different single coaxial apertures pierced into a metallic layer. For all, the metal thickness is set to $h=100 \mathrm{~nm}$ and the structure is supposed to be free standing (surrounded by vacuum). Dotted line corresponds to a PEC structure with $R_{o}=90 \mathrm{~nm}$ while the solid line corresponds to the same structure in silver. The dotdashed line and the dashed one are both calculated for $R_{o}$ $=125 \mathrm{~nm}$ and for PEC and silver metal, respectively. 
a $z$-finite structure is performed and the imaginary part of the effective index is determined by measuring the light attenuation. The schema of the studied structure is presented in Fig. 7.

A pulsed guided mode is injected at the point $I$. This pulse is centered around $\lambda=500 \mathrm{~nm}$ and has a small temporal width in order to cover the whole visible range. Three point detectors are set at $A, B$, and $C$. The three components $\left[E_{r}(t)\right.$, $E_{\phi}(t)$, and $\left.E_{z}(t)\right]$ of the electromagnetic field are then recorded versus time at these three points.

A time Fourier transform is then made over each component in order to determine the spectral densities $I_{A}, I_{B}$, and $I_{C}$.

Thus, the imaginary part of the effective index can be easily calculated from one of the following equations:

$$
n^{\prime \prime}=\frac{\lambda \ln \left(I_{A}, B \text { or } C / I_{B}, C \text { or } A\right)}{4 \pi\left(z_{B}, C \text { or } A-z_{A}, B \text { or } C\right)} .
$$

One notice here that this BOR FDTD calculation is time consuming (36 h on a laptop computer Dell Precision M70) because of the small spatial meshing in the radial direction $\left(\delta_{r}=0.1 \mathrm{~nm}\right)$ which leads to a very small time step in the FDTD code $\left(\delta t \simeq 1.35 \times 10^{-19} \mathrm{~s}\right)$ and also because of the weak value of the group velocity.

On the other hand, this method remains valid only for wavelengths less than the cutoff. In fact, for $\lambda=\lambda^{c}$, the group velocity of the guided mode is zero and the light does not propagate.

Because of this, Fig. 8 presents the logarithm of the imaginary part of the effective index only for $\lambda<\lambda^{c}$ for three different geometrical configurations. For all three cases $R_{i}$ is fixed to $75 \mathrm{~nm}$. The dotted line is obtained for $R_{o}=125 \mathrm{~nm}$, the dashed one for $R_{o}=250 \mathrm{~nm}$, and the solid one in the case of $R_{o}=80 \mathrm{~nm}$.

Figure 8 shows that the imaginary part of the lower guided mode (the $\mathrm{TE}_{11}^{\prime}$ one) increases when $R_{o}$ decreases, and then the decay length decreases. Moreover, $n_{\text {eff }}^{\prime \prime}$ is around $2 \times 10^{-3}$ for the whole visible range when $R_{o}=125 \mathrm{~nm}$. This corresponds to a decay length of $50 \mu \mathrm{m}$ (in average). Thus, this mode presents weak losses during its propagation and waveguides of several tens of micrometers can be designed for optical applications.

On the other hand, this finding can be used in the domain of enhanced transmission through subwavelength apertures as studied by Haftel et al. ${ }^{21}$ In that study, the authors demonstrate that by decreasing the value of the outer radius, the transmission peak is shifted toward large wavelength values. Figure 9 shows the zero-order efficiency of a 100-nm-thick silver layer perforated by only one aperture. Four apertures were studied: cylindrical and coaxial ones in PEC and in silver, and two different geometrical configurations.

Two phenomena are clearly shown on Fig. 9: first, the use of silver instead of PEC leads to a shift in the transmission peak toward a larger value of wavelength and, second, this shift can be amplified by decreasing the outer radius.

To the best of our knowledge, this work shows for the first time the determination of cutoff frequencies of a real metallic coaxial waveguide. In addition, the abnormal behavior of the cutoff wavelength of the fundamental mode is pointed out: it increases when the outer radius decreases. The group velocity of such a mode shows a weak value compared to $c$, which means that light will propagate slowly inside the waveguide. This property is very important if we consider nonlinear or electro-optical materials placed between the inner and the outer metallic parts because their coefficients can then be strongly increased with slow light.

This work is partly supported by the European Network of Excellence on Micro Optics NEMO (WP13). The grant of A.B. is funded by international collaboration between France and Algeria.
*FAX: 00 (33) 3816664 23. Electronic address: fbaida@univfcomte.fr

${ }^{1}$ M. Roussey, M.-P. Bernal, N. Courjal, and F. I. Baida, Appl. Phys. Lett. 87, 241101 (2005).

${ }^{2}$ H. Rigneault, J. Capoulade, J. Dinitnger, J. Wenger, N. Bonod, E. Popov, T. W. Ebbesen, and P. F. Lenne, Phys. Rev. Lett. 95, 117401 (2005).

${ }^{3}$ J. Wenger, H. Rigneault, J. Dintinger, D. Marguet, and P.-F. Lenne, J. Biol. Phys. 32, SN1 (2006).

${ }^{4}$ T. W. Ebbesen, H. J. Lezec, H. F. Ghaemi, T. Thio, and P. A. Wolff, Nature (London) 391, 667 (1998).

${ }^{5}$ F. I. Baida and D. Van Labeke, Opt. Commun. 209, 17 (2002).

${ }^{6}$ F. I. Baida and D. Van Labeke, Phys. Rev. B 67, 155314 (2003).

${ }^{7}$ F. I. Baida, Y. Poujet, B. Guizal, and D. Van Labeke, Opt. Commun. 256, 190 (2005).

${ }^{8}$ L. Novotny and C. Hafner, Phys. Rev. E 50, 4094 (1994).

${ }^{9}$ Ursula Schröter and Alain Dereux, Phys. Rev. B 64, 125420 (2001).
${ }^{10}$ Sergey I. Bozhevolnyi, Valentyn S. Volkov, Eloîse Devaux, and Thomas W. Ebbesen, Phys. Rev. Lett. 95, 046802 (2005).

${ }^{11}$ J. D. Jackson, Classical Electrodynamics, 2nd ed. (John Wiley, New York, 1975).

${ }^{12}$ P. B. Johnson and R. W. Christy, Phys. Rev. B 6, 4370 (1972).

${ }^{13}$ D. B. Davidson and R. W. Ziolkowski, J. Opt. Soc. Am. A 11, 1471 (1994).

${ }^{14}$ A. Taflove and S. C. Hagness, Computational Electrodynamics: The Finite-Difference Time-Domain Method, 2nd ed. (Artech House, Norwood, MA, 2000).

${ }^{15}$ C. T. Chan, Q. L. Yu, and K. M. Ho, Phys. Rev. B 51, 16635 (1995).

${ }^{16}$ F. I. Baida, D. Van Labeke, G. Granet, A. Moreau, and A. Belkir, Appl. Phys. B: Lasers Opt. 79, 1 (2004).

${ }^{17}$ Yong Xu, Reginald K. Lee, and Amnon Yariv, Opt. Lett. 27, 1019 (2002).

${ }^{18}$ Hocheol Shin, Peter B. Catrysse, and Shanhui Fan, Phys. Rev. B 72, 085436 (2005). 
${ }^{19}$ F. I. Baida, D. Van Labeke, and Y. Pagani, Opt. Commun. 255, 241 (2003).

${ }^{20}$ Reuven Gordon and Alexandre G. Brolo, Opt. Express 13, 1933 (2005).

${ }^{21}$ Michael I. Haftel, Carl Schlockermann, and Girsh Blumberg,
Appl. Phys. Lett. 88, 193104 (2006).

${ }^{22}$ Ruey-Lin Chern, Chien C. Chang, and C. Chung Chang, Phys. Rev. E 73, 036605 (2006).

${ }^{23}$ K. Sakoda, N. Kawai, T. Ito, A. Chutinan, S. Noda, T. Mitsuyu, and K. Hirao, Phys. Rev. B 64, 045116 (2001). 